\title{
A theorem on absolute summability of Fourier series by Riesz means
}

\section{Prem Chandra}

In 1951 Mohanty established the following theorem.

If $\phi(t) \log \log \frac{k}{t}$ is of bounded variation in $(0, \pi)$, where $k \geq \pi e^{2}$ and $\phi(t)=\frac{1}{2}\{f(x+t)+f(x-t)\}$, then $\sum_{n=1}^{\infty} A_{n}(x)$ is sumable $\left|R, \exp \left\{(\log w)^{\Delta}\right\}, I\right|$, for however large positive $\Delta$. In this present note we have generalised the above theorem by taking a more general type of Riesz means and under the condition, $\phi(t)\left(\log \log \frac{k}{t}\right)^{c}$ is of bounded variation in $(0, \pi)$, where $c$ is finite, imposed upon the generating function of Fourier series.

\section{Definitions and notations}

Let $L=L(w)$ be a differentiable, monotonic increasing function of $w$ tending to infinity with $w$. For a given infinite series $\sum a_{n}$, we write

$$
A_{p}(w)=\sum_{n \leq w}\{L(w)-L(n)\}^{r} a_{n} \quad(r \geq 0)
$$

The series $\sum a_{n}$ is summable $|R, L, r|(r>0)$ or symbolically $\sum a_{n} \in|R, L, r|(r>0)$, if

Received 21 May 1970. Communicated by S. Izumi.

179 


$$
\int_{h}^{\infty}\left|\frac{L^{\prime}(w)}{\{L(w)\}^{r+1}} \sum_{n \leq w}\{L(w)-L(n)\}^{r-1} L(n) a_{n}\right| d w
$$

is convergent, where $h$ is a positive number. (Obrechkoff [2], [3].)

Let $f(t)$ be a periodic function with period $2 \pi$ and integrable in the sense of Lebesgue over $(-\pi, \pi)$. We can, without any loss of generality, write the Fourier series of $f(t)$ as

$$
\sum_{n=1}^{\infty}\left(a_{n} \cos n t+b_{n} \sin n t\right)=\sum_{n=1}^{\infty} A_{n}(t)
$$

assuming that the constant term is zero.

Throughout we use the following notations:

$$
\begin{aligned}
& \text { (1.1) } \phi(t)=\frac{1}{2}[f(x+t)+f(x-t)\} ; \\
& \text { (1.2) } g(w, t)=\sum_{n \leq w} L(n) \cos n t(\log \log (n+2))^{c-1}, \quad(c \text { is finite); } \\
& \text { (1.3) } h(w, t)=\sum_{n \leq w} L(n) \sin n t / n(\log \log (n+2))^{1-c},(c \text { is finite). }
\end{aligned}
$$

\section{Introduction}

Concerning the absolute Riesz summability of Fourier series of order unity, Mohanty [1] proved the following:

THEOREM M. If $\phi(t) \log \log \frac{k}{t} \in B V(0, \pi), 1$ where $k \geq \pi e^{2}$, then $\sum_{n=1}^{\infty} A_{n}(x) \in\left|R, \exp \left\{(\log \omega)^{\Delta}\right\}, 1\right|$, for however large positive $\Delta$.

Generalising the above theorem, we prove the following:

THEOREM. Let $c$ be finite and $\Delta$ be positive however large. If the type of Riesz means $L(w)$ satisfies the following conditions:

$$
\left\{L(w) / w(\log \log w)^{1-c}\right\}
$$


is monotonic increasing with $w \geq w_{0}, 2$

$$
w L^{\prime}(w)=0\left\{L(w)(\log w)^{\Delta-1}\right\}
$$

Then, if $\phi(t)\left(\log \log \frac{k}{t}\right)^{c} \in B V(0, \pi)$, where $k \geq \pi e^{2}$, then

$\sum_{n=1}^{\infty} \frac{A_{n}(x)}{\{\log \log (n+2)\}^{1-c}} \in|R, L(w), 1|$.

\section{Proof of the theorem}

We have

$$
\begin{aligned}
A_{n}(x) & =\frac{2}{\pi} \int_{0}^{\pi} \phi(t) \cos n t d t \\
& =\frac{2}{\pi} \int_{0}^{\pi} \phi(t)\left(\log \log \frac{k}{t}\right)^{c} \frac{\cos n t}{\left(\log \log \frac{k}{t}\right)^{c}} d t \\
& =\frac{2}{\pi} \phi(\pi)\left(\log \log \frac{k}{\pi}\right)^{c} \int_{0}^{\pi} \frac{\cos n u}{\left(\log \log \frac{k}{u}\right)^{c}} d u \\
& -\frac{2}{\pi} \int_{0}^{\pi} d\left\{\phi(t)\left(\log \log \frac{k}{t}\right)^{c}\right\} \int_{0}^{t} \frac{\cos n u}{\left(\log \log \frac{k}{u}\right)^{c}} d u .
\end{aligned}
$$

Since $\phi(t)\left(\log \log \frac{k}{t}\right)^{c} \in B V(0, \pi)$, the series

$\sum_{n=1}^{\infty} \frac{A_{n}(x)}{\{\log \log (n+2)\}^{1-c}} \in|R, L(w), 1|$ if

$$
\begin{aligned}
I & =\int_{e}^{\infty} \frac{L^{\prime}(w)}{\{L(w)\}^{2}}\left|\int_{0}^{t} g(w, u)\left(\log \log \frac{k}{u}\right)^{-c} d u\right| d w \\
& =0(1),
\end{aligned}
$$

uniformly in $0<t \leq \pi$.

Integrating by parts, we have,

2 In the case that $\left\{L(w) / w(\log \log w)^{1-c}\right\}$ is monotonic decreasing, the result follows by using the second theorem of consistency for absolute Riesz summability. 
$\int_{0}^{t} \frac{g(w, u)}{\left(\log \log \frac{k}{u}\right)^{c}} d u=\frac{h(w, t)}{\left(\log \log \frac{k}{t}\right)^{c}}+c \int_{0}^{t} \frac{h(w, u)}{u\left(\log \frac{k}{u}\right)\left(\log \log \frac{k}{u}\right)^{1+c}} d u$

Therefore

$I \leq\left(\log \log \frac{k}{t}\right)^{-c} \int_{e}^{\infty} \frac{L^{\prime}(w)}{\{L(w)\}^{2}}|h(w, t)| d w$

$$
+\int_{e}^{\infty} \frac{L^{\prime}(w)}{\{L(w)\}^{2}}\left|\int_{0}^{t} \frac{c^{-1} h(w, u)}{\log \frac{k}{u}\left(\log \log \frac{k}{u}\right)^{1+c}} d u\right| d w
$$

$=I_{1}+I_{2}$, say.

Now

$$
\int_{0}^{t} \frac{\sin n u}{u \log \frac{k}{u}\left(\log \log \frac{k}{u}\right)^{1+c}} d u=0\left\{(\log (n+1))^{-1}(\log \log (n+2))^{-(1+c)}\right\}
$$

we have

$$
\begin{aligned}
I_{2} & =0\left\{\int_{e}^{\infty} \frac{L^{\prime}(w)}{\{L(w)\}^{2}}\left|\sum_{n \leq w} \frac{n^{-1} L(n)}{\log (n+1)(\log \log (n+2))^{2}}\right| d w\right\} \\
& =0(1),
\end{aligned}
$$

since

$$
\begin{gathered}
\qquad \sum_{n=1}^{\infty}(n \log (n+1))^{-1}(\log \log (n+2))^{-2}<\infty . \\
\text { For } T_{1}=k / t \text { and } T_{2}=(k / t)\left(\log \frac{k}{t}\right)^{\Delta-1} \text {, we write } \\
\qquad \begin{aligned}
I= & \left(\log \log \frac{k}{t}\right)^{-c}\left(\int_{e}^{T_{1}}+\int_{T_{1}}^{T_{2}}+\int_{T_{2}}^{\infty}\right)\left(\frac{L^{\prime}(w)}{\{L(w)\}^{2}}|h(w, t)| d w\right) \\
= & I_{1,1}+I_{1,2}+I_{1,3}, \text { say. }
\end{aligned}
\end{gathered}
$$

By using the fact $\mid$ sinnt $\mid \leq n t$, we have 


$$
\begin{aligned}
I_{1,1} & \leq \frac{t}{\left(\log \log \frac{k}{t}\right)^{c}} \int_{e}^{T_{1}} \frac{L^{\prime}(x)}{\{L(w)\}^{2}}\left|\sum_{n \leq w} \frac{L(n)}{\{\log \log (n+2)\}^{1-c}}\right| d w \\
& =0\left\{t\left(\log \log \frac{k}{t}\right)^{-c} \int_{e}^{T_{1}} \frac{L^{\prime}(w)}{\{L(w)\}^{2}} d w \int_{1}^{w} \frac{L(x)}{\{\log \log (x+2)\}^{1-c}} d x\right\}+0(1) \\
& =0\left\{t\left(\log \log \frac{k}{t}\right)^{-c} \int_{I}^{T}\{\log \log (x+2)\}^{c-1} d x\right\}+0(1) \\
& =0(1),
\end{aligned}
$$

uniformly in $0<t \leq \pi$; end using sinnt $=0(1)$, we have

$$
\begin{aligned}
I_{1,2} & =0\left\{\left(\log \log \frac{k}{t}\right)^{-c} \int_{T_{1}}^{T_{2}} \frac{L^{\prime}(w)}{\{L(w)\}^{2}} d w \int_{1}^{w} \frac{x^{-1} L(x)}{\{\log \log (x+2)\}^{1-c}} d x\right\}+o(1) \\
& =0\left\{\left(\log \log \frac{k}{t}\right)^{-c} \int_{T_{1}\{\log \log (x+2)\}^{1-c}}^{T_{2}} \frac{x^{-1}}{\{x\}+0(1)}\right. \\
& =0\left\{\left(\log \log \frac{k}{t}\right)^{-1}\left(\log T_{2}-\log T_{1}\right)\right\}+0(1) \\
& =0(1),
\end{aligned}
$$

uniformly in $0<t \leq \pi$.

Since, by applying Abel's Lemma, in view of (2.1),

$$
h(w, t)=o\left\{t^{-1} L(w) / w(\log \log w)^{1-c}\right\},
$$

we have

$$
\begin{aligned}
& I_{1,3}=0\left\{t^{-1}\left(\log \log \frac{k}{t}\right)^{-c} \int_{T_{2}}^{\infty} \frac{w^{-1} L^{\prime}(w)}{L(\omega)(\log \log w)^{1-c}} d w\right\} \\
& =o\left\{t^{-1}\left(\log \log \frac{k}{t}\right)^{-c} \int_{T_{2}}^{\infty} \frac{w^{-2}(\log w)^{\Delta-1}}{(\log \log w)^{1-c}} d \omega\right\} \text { (by (2.2)) } \\
& =0(1) \text {, }
\end{aligned}
$$

uniformly $0<t \leq \pi$.

This terminates the proof of the theorem. 


\section{References}

[1] R. Mohanty, "On the absolute Riesz summability of Fourier series and allied series", Proc. London Math. Soc. (2) 52 (1951), 295-320.

[2] Nicolas Obrechkoff, "Sur la sommation absolue des séries de Dirichlet", C.R. Acad. Sci. Paris 186 (1928), 215-217.

[3] Nikola Obreschkoff, "Über die absolute Sumierung der Dirichletschen Reihen", Math. 2. 30 (1929), 375-385.

Government Science College, Jabalpur, India. 